\title{
The First Billion Years simulation project. Galactic outflows and metal enrichment
}

\author{
Claudio Dalla Vecchia, ${ }^{1, a}$ Sadegh Khochfar ${ }^{1, b}$ and Joop Schaye ${ }^{2}$ \\ ${ }^{1}$ Max Planck Institute for Extraterrestrial Physics, \\ Giessenbach-Straße, D-85748, Garching, Germany \\ emails: a) caius@mpe.mpg.de; b) sadeghk@mpe.mpg.de \\ ${ }^{2}$ Leiden Observatory, University of Leiden, \\ Niels Bohrweg 2, NL-2333CA, Leiden, The Netherlands \\ email: schaye@strw.leidenuniv.nl
}

\begin{abstract}
The First Billion Years is one of the largest and most comprehensive cosmological, hydrodynamical simulation of the early Universe to date. It studies the formation of the first proto-galaxies down to redshift 6 , and provides the path of the evolution from proto-galaxies to the observed galaxy population at redshift 4 . It also provides predictions for future observations.
\end{abstract}

Keywords. galaxies: evolution, galaxies: formation, galaxies: high-redshift, galaxies: ISM, methods: numerical

\section{Introduction}

In the last decade, deep observations of the Universe have been providing high quality data in the redshift range $4<z \lesssim 6$. In the near future, the James Webb Space Telescope will be probing even higher redshifts with observations of proto-galaxies formed when the Universe was less that one billion years old. Providing a detailed, theoretical framework of the formation of these proto-galaxies will help to predict, as well as to interpret, what will be observed. The First Billion Years simulation project (FiBY) has been conducted with this aim.

$F i B Y$ is one of the largest and most comprehensive cosmological, hydrodynamical simulation of the early Universe to date. A volume of $(8 \mathrm{Mpc})^{3}$ containing $2 \times 1368^{3}$ dark matter and gas particles is evolved down to redshift $z=6$. The gas mass resolution is $1250 \mathrm{M}_{\odot}$, whereas the spatial resolution is a few tens of physical parsecs. Such high resolution is necessary to resolve the collapse of the first structures - the mini-haloes that host the first generation of stars (population-III stars, hereafter pop-III).

The collapse of gas of primordial composition into mini-haloes is driven by molecular hydrogen cooling. This gas is the fuel for the formation of pop-III stars. These stars are responsible for the early enrichment of the inter-galactic medium (IGM) through very energetic pair-instability supernova (PISN) explosions, creating the conditions for the formation of the metal-enriched, second generation stars (population-II stars, hereafter pop-II). Therefore, molecular formation and molecular cooling, pop-III and pop-II star formation and stellar feedback are the main ingredients for building the first galaxies.

A set of simulations with increasing volume and decreasing resolution have been performed to provide a larger range of galaxy masses, to study the effect of numerical resolution, and to test variations of the physics and parameters in the model. When combined, these simulations extend the low mass end of the simulated galaxy mass function down to $10^{5} \mathrm{M}_{\odot}$. This corresponds to resolving galaxies with masses two orders of magnitude smaller than in simulations that are currently state-of-the-art (e.g. Finlator et al. 2011). 
Table 1. Summary of simulation parameters: simulation name; co-moving box length, $L$; number of particles, $N_{\text {tot }}$; gas particle mass, $m_{\mathrm{b}}$; dark matter particle mass, $m_{\mathrm{DM}}$; co-moving gravitational softening, $\epsilon$.

\begin{tabular}{cccccc}
\hline Simulation & $\begin{array}{c}L \\
\left(h^{-1} \mathrm{Mpc}\right)\end{array}$ & $N_{\text {tot }}$ & $\begin{array}{c}m_{\mathrm{b}} \\
\left(h^{-1} \mathrm{M}_{\odot}\right)\end{array}$ & $\begin{array}{c}m_{\mathrm{DM}} \\
\left(h^{-1} \mathrm{M}_{\odot}\right)\end{array}$ & $\begin{array}{c}\epsilon \\
\left(h^{-1} \mathrm{kpc}\right)\end{array}$ \\
\hline FiBY08XL & 5.68 & $2 \times 1368^{3}$ & $8.87 \times 10^{2}$ & $4.36 \times 10^{3}$ & 0.17 \\
\hline FiBY04M & 2.84 & $2 \times 684^{3}$ & $8.87 \times 10^{2}$ & $4.36 \times 10^{3}$ & 0.17 \\
FiBY08M & 5.68 & $2 \times 684^{3}$ & $7.10 \times 10^{3}$ & $3.48 \times 10^{4}$ & 0.33 \\
FiBY16M & 11.36 & $2 \times 684^{3}$ & $5.68 \times 10^{4}$ & $2.79 \times 10^{5}$ & 0.66 \\
FiBY32M & 22.72 & $2 \times 684^{3}$ & $4.54 \times 10^{5}$ & $2.23 \times 10^{6}$ & 1.33 \\
\hline
\end{tabular}

The list of FiBY simulations is in Table 1. A brief description of the code employed in the project is in the next section.

\section{Numerical tool}

The code used for the project is an extension of that employed in the OWLS project (Schaye et al. 2010). The $O W L S$ code is a modified version of GADGET (Springel 2005) with a novel treatment of star formation (Schaye \& Dalla Vecchia 2008), stellar evolution (Wiersma et al. 2009b), metal line cooling (Wiersma et al. 2009a) and black hole feedback (Booth \& Schaye 2009). It was updated by introducing molecular hydrogen formation and cooling, gas shielding from the UV background (e.g. Nagamine et al. 2010), pop-III star formation and feedback, and stellar dust yields. Stellar feedback has been improved by adopting the model of Dalla Vecchia \& Schaye (2012), where thermal energy from SN explosions is injected in the surrounding gas. As shown by these authors, the effect

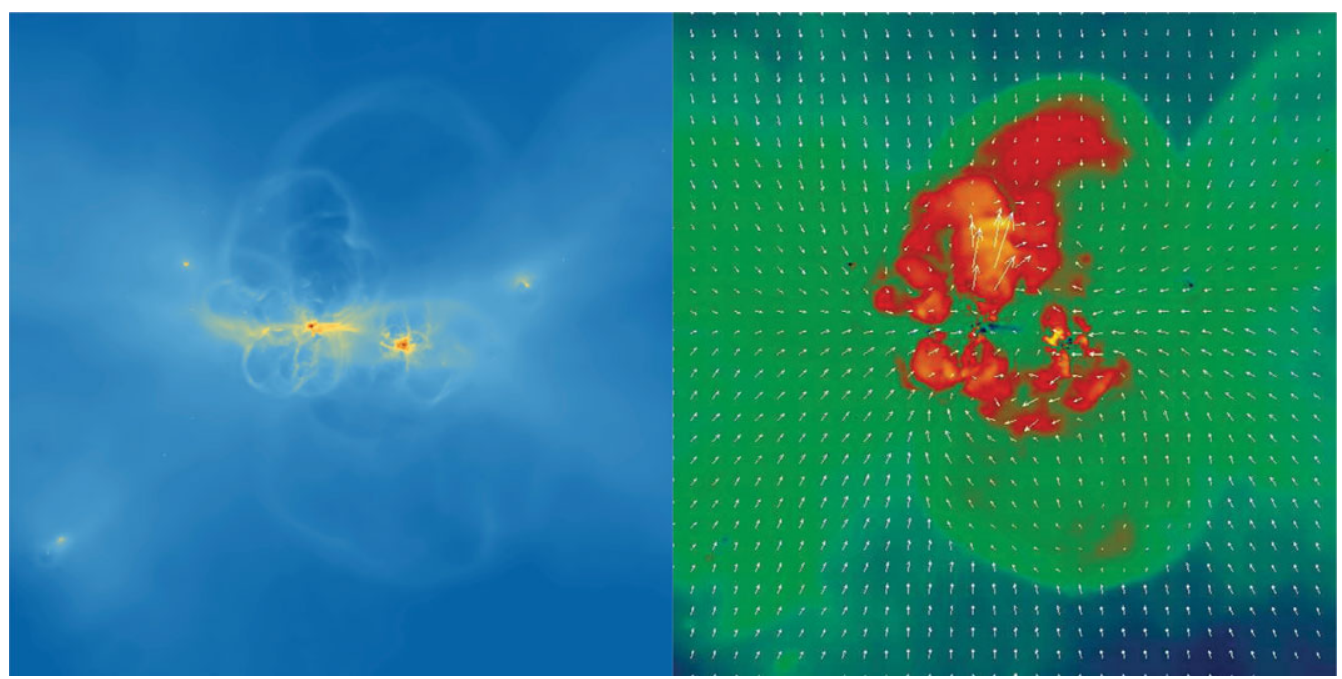

Figure 1. Projected density (left panel) and mass-weighted temperature (right panel) maps of a box of 900 co-moving kpc centred on the most massive halo in FiBY04M. The colour scale is logarithmic. Two interacting galaxies are in a filament which is horizontal in the picture. Several shock fronts surrounding underdense bubbles are clearly visible in the density map and are the result of several stellar feedback episodes. The temperature of the gas inside the bubbles (right panel) is larger than the background temperature and can be as high as $10^{5-6} \mathrm{~K}$. The over-plotted velocity field shows the accretion along the filament as well as the galactic outflow. 

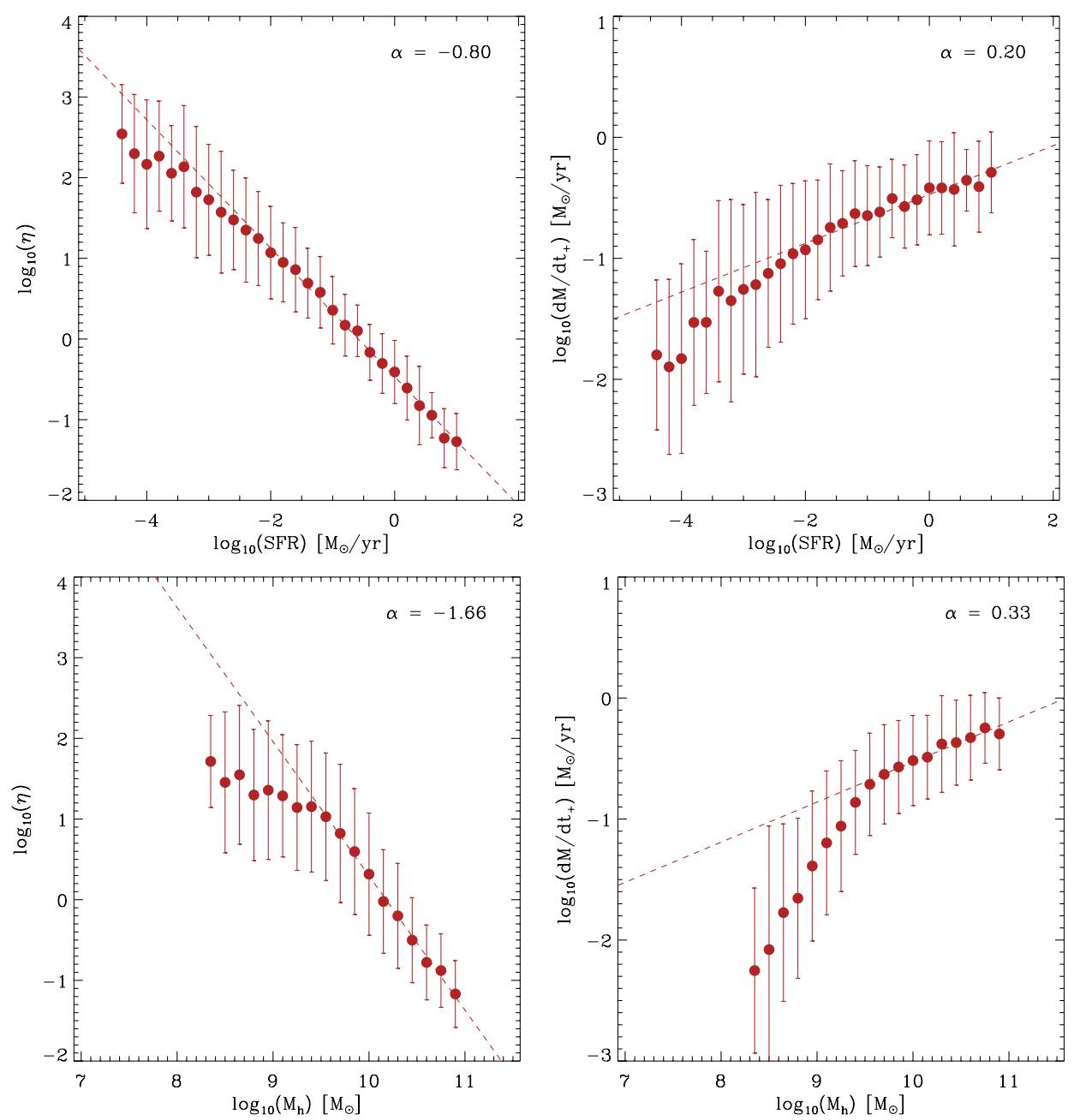

Figure 2. Outflow mass loading (left panels), $\eta$, and rate (right panels) as function of star formation rate (top row) and halo mass (bottom row). The dashed lines are fits of the bin averages for halo masses $M_{\mathrm{h}}>10^{9.5} \mathrm{M}_{\odot}$. The slope of the fitting relations is reported in the top-right corner of each plot. The break in the relations is evident when plotting the values against halo mass.

of feedback depends on resolution. The resolution criterion they propose is matched in all but the largest volume simulations. The effect of feedback in creating large galactic outflows is depicted in Fig. 1. Hot and metal-rich bubbles extend well into the IGM contributing to the enrichment of a large volume. The metal volume-filling factor at $z=6$ is $\sim 10^{-3}$ (Johnson et al. 2013).

\section{Preliminary results}

Fig. 2 shows outflow properties as function of star formation rate (SFR) and halo mass. The mass loading (the ratio between mass outflow rate and SFR), $\eta$, is plotted in the left panels, whereas the mass outflow rate is plotted in the right panels. All gas particles at the virial radius that are moving with (positive) radial velocity larger than the halo 
escape velocity are considered to be outflowing. There is a clear break in the relations at halo masses $M_{\mathrm{h}} \simeq 10^{9.5} \mathrm{M}_{\odot}$. This is not present in the galaxies mass and luminosity functions which match the observed data at $z \sim 6$. Therefore, the stellar mass of haloes does not seem to play a role.

What plays a role in defining the break is the stellar feedback. Feedback is more effective in removing gas from small haloes. Haloes in a low density environment cannot accrete gas efficiently, therefore, after feedback has removed a large part of their gas content, their stellar mass does not grow further. This becomes clear when plotting the ratio of stellar mass to gas mass, $f_{*}=M_{*} / M_{\mathrm{g}}$, where, for $M_{\mathrm{h}}<10^{9.5} \mathrm{M}_{\odot}$, the ratio flattens. The flattening of $f_{*}$ is in the numerically resolved mass range.

Okamoto et al. (2008) showed that the same characteristic halo mass (the so called filtering mass) is imprinted by re-ionisation processes, and galaxies in halos smaller than $10^{9.5} \mathrm{M}_{\odot}$ cannot grow efficiently. However, in $F i B Y$, the flattening of $f_{*}$ is observed well before re-ionisation happens in the simulations. The conclusion is that stellar feedback may be the main driver defining the filtering mass at any redshift before re-ionisation.

\section{References}

Booth, C. M. \& Schaye, J. 2009, MNRAS, 398, 53

Dalla Vecchia, C. \& Schaye, J. 2012, MNRAS, 426, 140

Finlator, K., Oppenheimer, B. D., \& Davé, R. 2011, MNRAS 410, 1703

Johnson, J. L., Dalla Vecchia, C., \& Khochfar, S. 2013, MNRAS, 428, 1857

Nagamine, K., Choi, J.-H., \& Yajima, H. 2010, ApJ, 725, L219

Okamoto, T., Gao, L., \& Theuns, T. 2008, MNRAS, 390, 920

Schaye, J., Dalla Vecchia, C., Booth, C. M., et al. 2010, MNRAS, 402, 1536

Schaye, J. \& Dalla Vecchia, C. 2008, MNRAS, 383, 1210

Springel, V. 2005, MNRAS, 364, 1105

Wiersma, R. P. C., Schaye, J., \& Smith, B. D. 2009, MNRAS, 393, 99

Wiersma, R. P. C., Schaye, J., Theuns, T., Dalla Vecchia, C., \& Tornatore, L. 2009, MNRAS, 399,574 